\title{
Detection of Interfaces And Voids in Pipelines Using Gamma Scanning
}

\author{
Suma T*, Yelgaonkar V. N. ", Tiwari C.B. ", Dhakar V.D. \\ * Scholar, Sri Satya Sai University of Technology and Medical Sciences, Sehore, Madhya Pradesh, India, \\ ${ }^{\#}$ Board of Radiation and Isotope Technology, Vashi, Navi Mumbai, India, 400705.
}

\begin{abstract}
Industrial activity involves plenty of technical problems to which solutions are sought at various levels, from trouble shooting to complete investigation and modeling of processes or plants, or development of new technologies. The purpose of this investigation is to apply the gamma scanning technique to detect the interface, voids present in pipe lines. The results of experiments performed in a leading petrochemical complex are presented. Gamma scanning of the pipeline carrying para xylene was carried out in three different orientations of the pipe line using Cesium-137 and the transmitted radiation intensity was recorded by using Scintillation detector $\mathrm{NaI}(\mathrm{Tl})$. Interface location and presence of voids in some of the bends were successfully identified using this technique. The interface locations observed were not found to be stable but oscillating up and down with some well-defined period of oscillation.
\end{abstract}

Keywords: Gamma scanning technique, Cesium-137, Scintillation detector, Interface, voids.

\section{Introduction}

In any industry, good plant performance and avoiding a shutdown, especially in continuous process, are of special importance for healthy production, economy and safety. Gamma scanning can provide speedy solutions to many plant and process problems, starting from malfunctions in specific components to poor overall plant performance. Performance of an industrial process depends upon chemical process optimization and intended fluid transfer. Fluid transfer takes place through pipelines of different sizes, configuration and analogy. Any trouble in fluid flow through the pipeline may lead to reduction in production resulting in revenue loss. The application of gamma pipe scanning has developed appreciably over the last few years. Gamma pipe scanning offers information that cannot be obtained by means of conventional methods. This technique is safe if all necessary precautions are taken. Gamma scanning technique is a very useful on-line troubleshooting tool to identify presence of liquid, solid and void inside the pipeline without disturbing the process. Gamma scanning provides a non-destructive and cost effective way of analyzing problems related to solid deposition, presence of voids and presence of foreign objects in the pipeline.

\section{Gamma Pipe Scanning}

When two different materials of dissimilar densities are present in tanks or in vessels, the location of interface/level of liquid could be identified exactly using gamma ray attenuation technique. When gamma ray emitted by a collimated radiation source on one side of the vessel/tank/pipe passes though the material in the vessel/tank/pipe, its extent of stoppage (attenuation) is inversely and exponentially proportional to the density of material. A sudden change in transmitted intensity will be observed when gamma rays pass from material at

higher density to the material at lower density or vice a versa. This change in transmitted radiation intensity from a source on one side of the vessel/tank/pipe is accurately monitored using highly sensitive radiation detector moving on the opposite side of the vessel/tank/pipe. Radioisotope Cesium-137 has suitable radiation energy to get clear contrast, reasonably long half-life to be used for such several applications in the years to come and possibility of economic production at high specific activity. Therefore it was decided to use the same for this investigation.

\section{Advantages And Disadvantages Of Pipe Scanning}

Major advantage of the pipe scan is its utility without disturbing the process i.e. it is carried out online. Conventional methods like pressure drop studies, sampling etc. can provide rough idea about the problem in the pipeline but they cannot pinpoint the problem area. Whereas, gamma pipe scan provides detailed insight of the pipeline and visualization of what could be happening inside the pipeline.

Disadvantage of this method is, it requires trained manpower, additional training for handling of radioisotopes and knowledge of radiation safety. 


\section{Safety Issues}

Usually radioisotope used for gamma pipe scan is Cesium-137. This has half-life of 33 years and emits gamma energy of $0.66 \mathrm{MeV}$. Intensity of the source used was $100 \mathrm{mCi}$ which is sufficient to scan the pipes of the diameters of about 36 inches. The source was collimated using tungsten collimator so as to get the concentrated beam of radiation of $2 \mathrm{~mm}$ width. However, this intensity will not be as hazardous as the intensity of a radiography source which is usually of the order of $10 \mathrm{Ci}$. In case of radiography being carried out the area surrounding needs to be cordoned off.

In general, criteria of time distance and shielding was observed while handling radioisotopes. Time taken to handle the radioisotope or when in vicinity was critically least minimum ( $<30$ seconds), the radioisotope was handled from the distance as far as possible. Care was taken to keep persons away from the source and the radioisotope was adequately shielded for handling i.e. surface dose rate on the container was less than $10 \mathrm{mR} /$ hour at any given time.

All of these factors were strictly taken in to account while carrying out the gamma pipe scanning. The radioisotope was handled by the trained and experienced professionals, it was maneuvered in the lead shielded collimators in the pipe scanner along the pipeline and it was observed that general public / plant staff and workers do not go in the vicinity of the source except those involved in the direct scanning.

\section{Experimental Procedure}

\subsection{Principle Of Gamma Pipe Scanning}

A schematic representation of the experimental arrangement is given in the above figure (Fig1). The transmission of $\gamma$ radiation through any material is governed by the exponential equation

Where:

$$
\mathbf{I}=\mathbf{I}_{0} \mathbf{e}^{-\mu x}
$$

I $\rightarrow$ Intensity of radiation transmitted through a material of thickness $\mathrm{Xcm}$

Io $\rightarrow$ Intensity of radiation from the $\gamma$ - ray source reaching the detector in the absence of the material.

$\mu \rightarrow$ Linear absorption coefficient $\left(\mathrm{cm}^{-1}\right)$

A collimated source and detector system is positioned diametrically opposite to the pipeline. The transmitted radiation, after traversing through the pipeline is detected by a collimated detector. The source and detector are moved along the length of the pipeline in the same horizontal plane. The transmitted radiation will be proportional to the density and thickness of material in the pipeline. After assessing the increase/decrease in the radiation intensity, the location of interface can be estimated.

\subsection{Gamma Pipe Scanning In The Pipeline}

The layout of the pipeline scanned is shown in Fig.2 The gamma scanning of the pipeline was carried out using radioactive source Cesium-137 (Cs-137), 100mCi. The energy of gamma rays of Cs-137 is $662 \mathrm{keV}$ which is sufficient for scanning pipelines up to 36". The source was placed in a lead collimator.

Scintillation detector $\mathrm{NaI}(\mathrm{Tl}), 1$ "xl", in conjunction with battery operated count rate meter was used to record the transmitted radiation intensity across the pipeline. The detector was placed in a lead collimator and placed diametrically opposite to the source. The schematic representation of the position of source and detector (Fig.3). Readings were obtained at $150 \mathrm{~mm}$ intervals.

\subsection{Describtion Of The Pipelines :}

The description of the pipelines being scanned is tabulated in Table 1.

\begin{tabular}{|l|l|l|l|l|l|l|}
\hline Exchanger & Line Size(inch) & Line ID(mm) & $\begin{array}{l}\text { Pipe thickness } \\
(\mathrm{mm})\end{array}$ & $\begin{array}{l}\text { Insulation } \\
\text { thickness (mm) }\end{array}$ & $\begin{array}{l}\text { Density } \\
\mathrm{Kg} / \mathrm{m}^{3}\end{array}$ & $\begin{array}{l}\text { Vertical } \\
\text { length of leg }(\mathrm{m})\end{array}$ \\
\hline E5-304 & $\begin{array}{l}32 \text { (top part) } \\
36 \text { (bottom part) }\end{array}$ & $\begin{array}{l}782.8(\text { top part) } 880.4 \\
\text { (bottom part) }\end{array}$ & $\begin{array}{l}15 \text { (top part) } \\
17 \text { (bottom part) }\end{array}$ & 165 & 867.9 & 24.009 \\
\hline E5-305 & 20 & 488 & 10 & 115 & 902.5 & 18.423 \\
\hline E5-306 & 12 & 311.2 & $6.35(\mathrm{SCH} 20)$ & $90(\mathrm{max})$ & 928.8 & 18.23 \\
\hline E5-307 & 10 & 260.4 & $6.35(\mathrm{SCH} 20)$ & $25(\mathrm{max})$ & 942.2 & 18.843 \\
\hline E5-308 & 10 & 257.4 & $7.8(\mathrm{SCH} 30)$ & $25(\max )$ & 994.1 & 14.415 \\
\hline
\end{tabular}

Table 1: The description of the pipelines being scanned 


\subsection{Interface Detection:}

\section{Results And Discussion}

a) For HX 304: Interface location is at about 402" from the HX bottom. The variation in readings in the higher side (vapour phase) represent statistical fluctuations. After the interface location, the liquid phase co-exists with some vapour coming from the bottom i.e. 2 phase flow. However, grossly it could be considered that the interface is located at 402". (Fig.4)

b) For HX 305: Interface appears to be dynamic due to the flow of liquid from top to bottom and flow of vapour from bottom to top. This is evident from the appearance of interface at 2 locations. Within the time span of scanning the one portion the interface has travelled to the other location. Hence it could be considered at 240" from the bottom of the HX. (Fig.5)

c) For HX 306: Interface location is identified at 312" from the HX bottom. The interface in this particular pipe is clearly noticed. Vapour portion in the upper part has fluctuations within statistical limits. (Fig.6)

d) For HX 307: No clear cut interface could be observed in this pipe. Some fluctuations are observed which could be due to the presence of sparged vapours which slightly change the dynamic density of the liquid phase. Otherwise it appears that there could be only liquid phase in this pipe. If the count-rate readings for reference pipeline are compared with those in this pipeline, it corroborates the indication. (Fig.7)

e) For HX 308: Interface could not be observed in this pipe. Consistency in the readings shows that a single medium of uniform density is present between the source and detector. (Fig.8)

f) The change in position of the interface with respect to time was observed in few pipes. The period of oscillations of interfaces in these pipes were measured and shown in Fig.9 to Fig.16.

\subsection{VOIDS:}

In few of the bends, the voids are not observed which could be due to smooth liquid phase flow. However, in many of the bends few or multiple voids are detected which could have been due to presence of vapour pockets. Sometimes these pockets may be static or sometimes dynamic with very slow speed. Thus, in case of doubts, repetition performed has also concurred with the same readings. Dimensions of the larger voids which could grossly affect the flow are mentioned in the figures. (Fig.17 to Fig.23)

\section{Conclusions}

In all the pipes mentioned above, starting from bottom of the heat exchangers stable interface could not be observed may be due to the existence of two dynamic phases together. It was suspected that the interface formed between vapour phase and liquid phase is not static and stationed at one location but it is oscillating up and down with some well-defined period of oscillation. This was monitored by placing source and detector stationed at suspected interface location for about 3 to 5 cycles i.e. between 9 to 15 minutes. The readings of count rate are plotted with respect to real time and the period of oscillation was monitored. Oscillations with specific period are observed in three pipelines viz. 304, $305 \& 306$. However, in 307 and 308 the readings remained almost constant and the variations are within the statistical fluctuations. The periodic oscillations could be due to the intermittent condensations in the heat exchangers and liquid formed is delivered through the headers towards the feed line. In these headers $(307 \& 308)$ liquid could be filled up to the monitoring level which is evident from the steady state count-rate observed in the Para xylene pipe compared with these pipes. There are voids present in some of the bends which could be due to vapour trapped and localized at a particular location which may be obstructing the smooth flow of fluid through the pipe.

\section{Acknowledgements}

We are deeply indebted to RIL Industries Ltd. Manufacturing division, Dehej, Gujarat, India who has supported our field staff during the work and providing necessary facilities for smooth functioning during the work period. It is worth acknowledging the fruitful tele conference with all the senior and top managers of the petrochemical complex where the experiments were conducted.

\section{References}

[1]. Charlton, J.S., 1986.RadioisotopeTechniques for Problem Solving in Industrial

[2]. Process Plants.LeonardHill.

[3]. Guidebook on radioisotope tracers in industry, 1990.Technical report Series

[4]. No. 316.InternationalAtomicEnergyAgency (IAEA), Vienna.

[5]. Zitny, R., Thyn, J., 1996.Programs For Residence Time distribution analysis, RTD

[6]. Software manuals for data analysis of radiotracer experiments. 


\section{Figures}

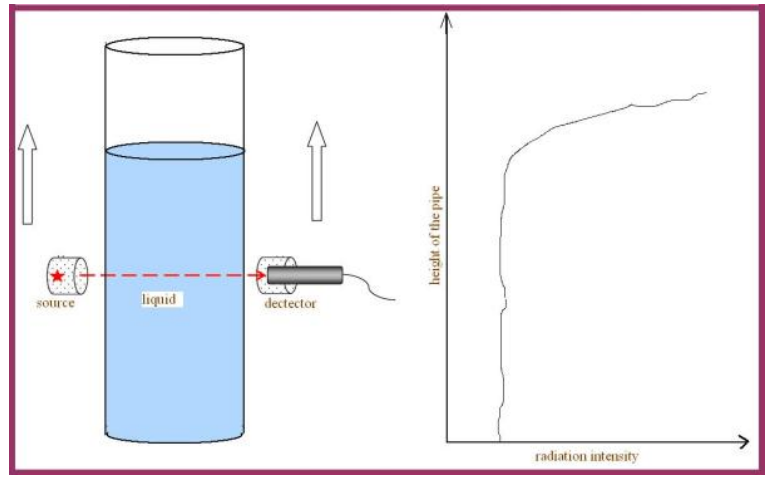

Fig 1: Interface detection/level measurement using gamma scanning

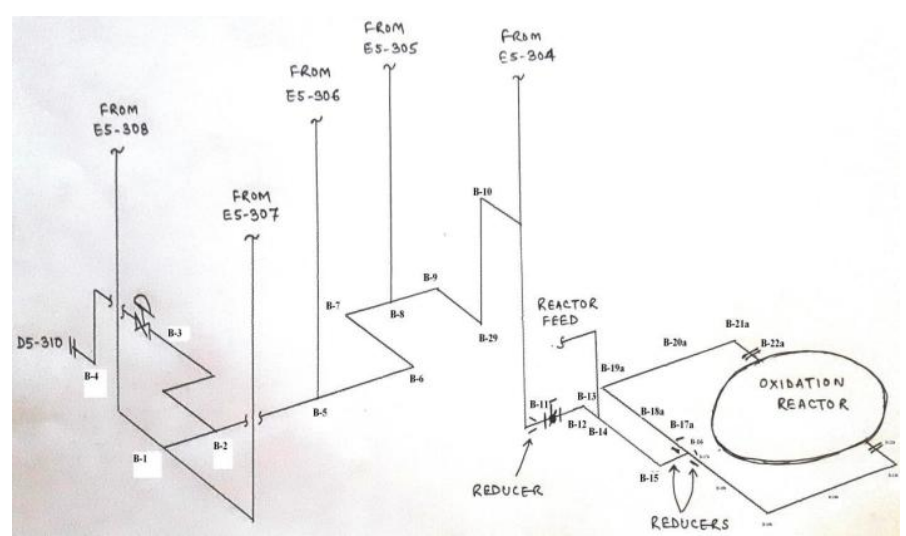

Fig 2: layout of the pipe line for gamma scanning
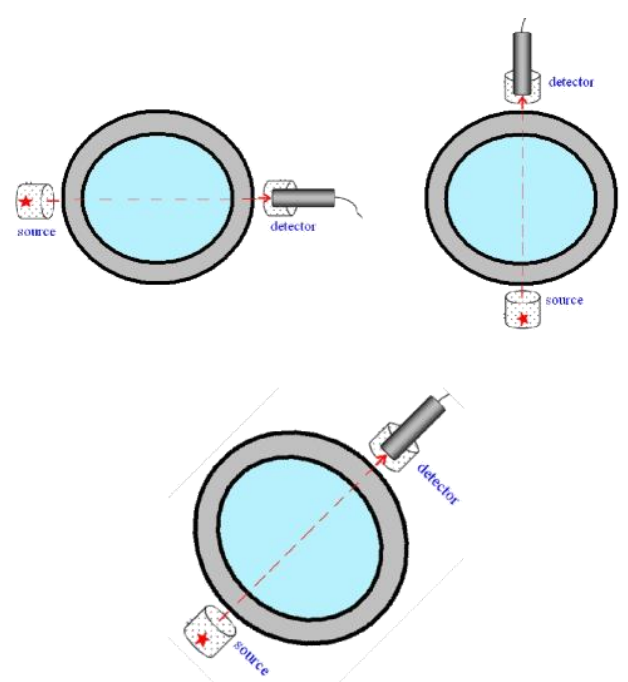

Fig 3: Orientations for gamma pipe scanning in $x, y$ and 


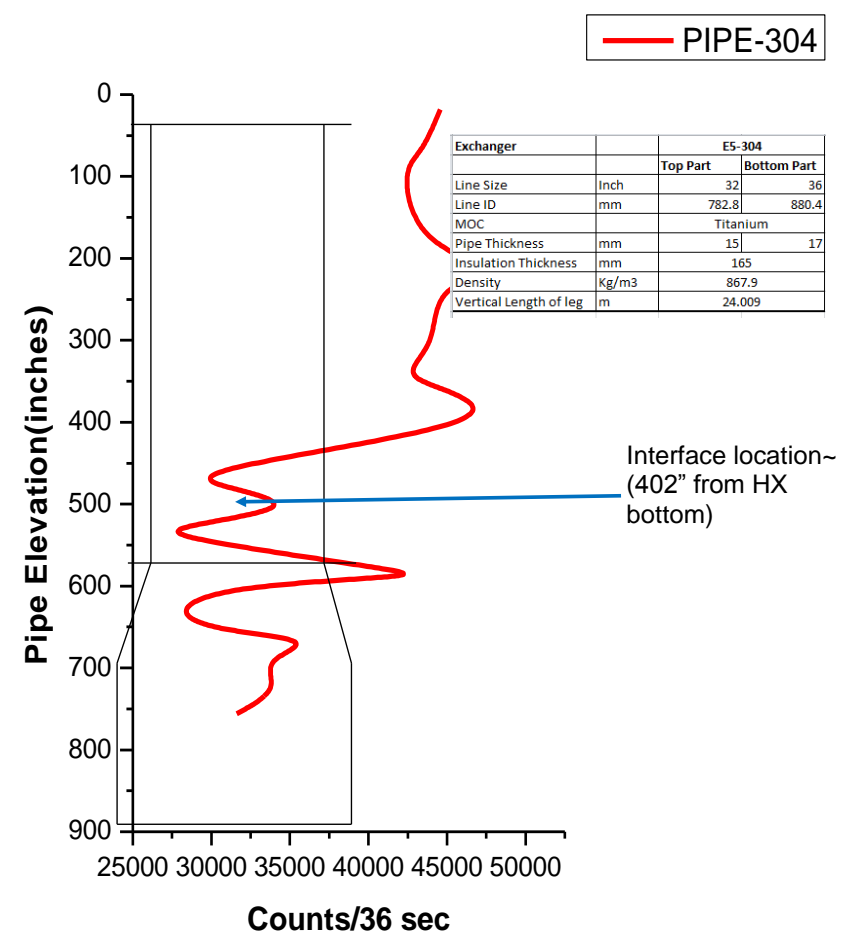

Fig 4: Interface in pipe 304

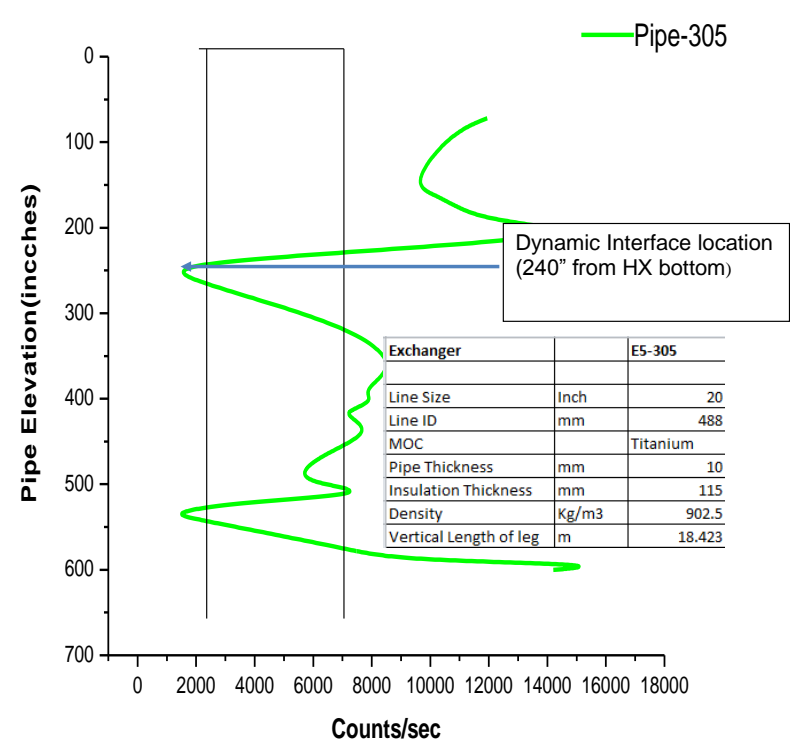

Fig 5:Interface in pipe 305 


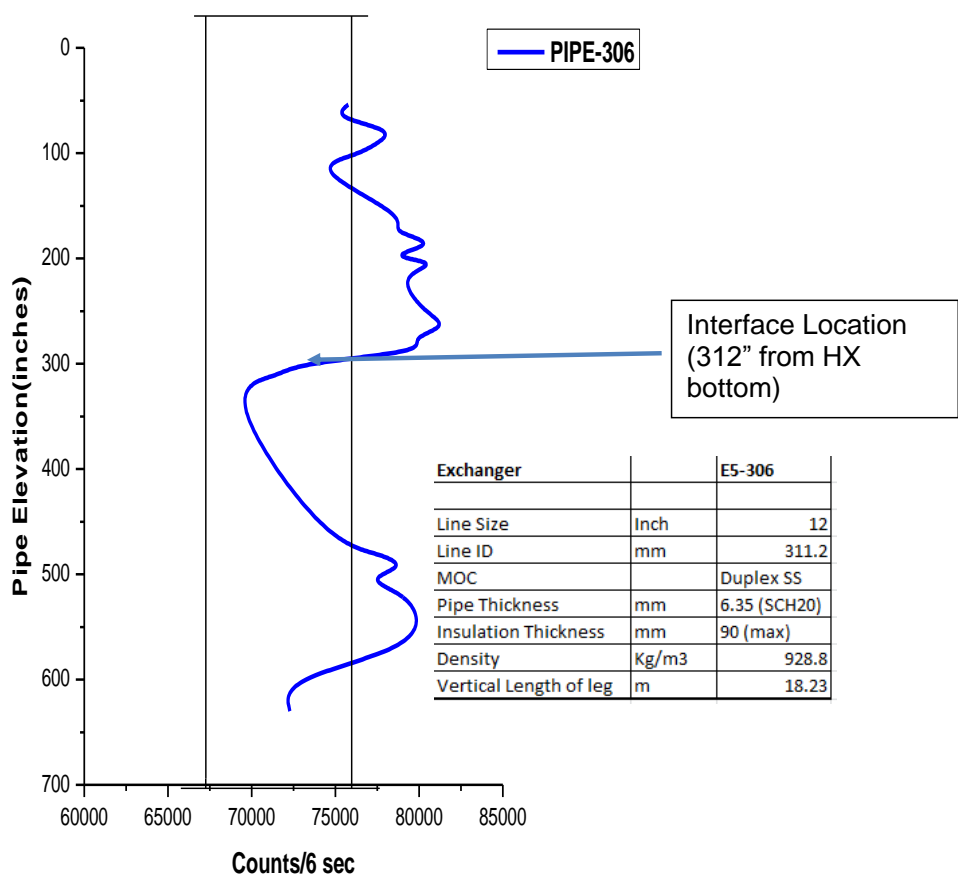

Fig 6: Interface in pipe 306

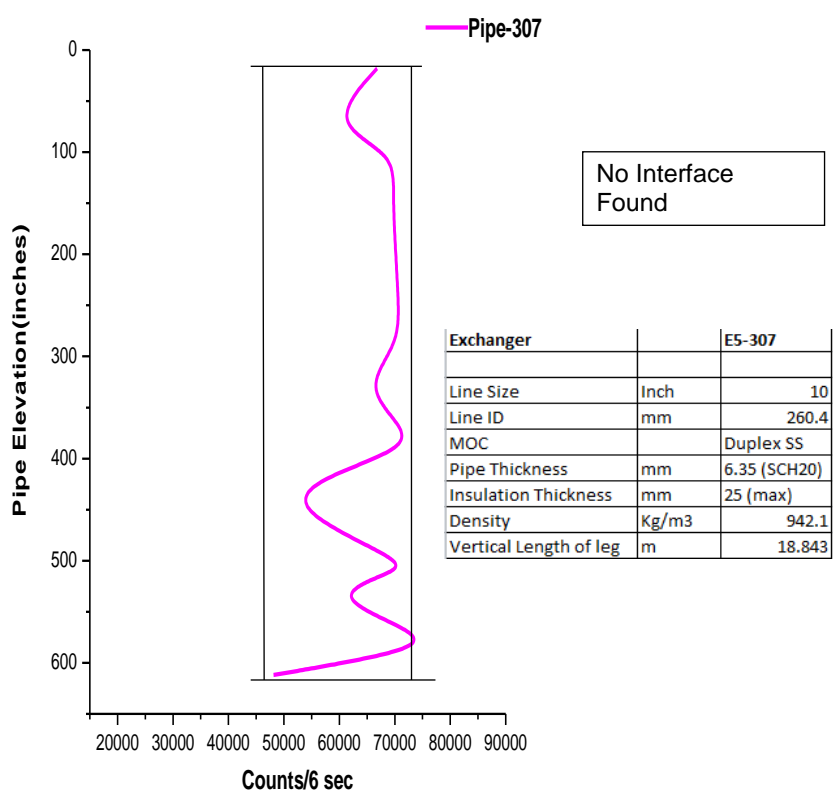

Fig 7: Interface in pipe 307 


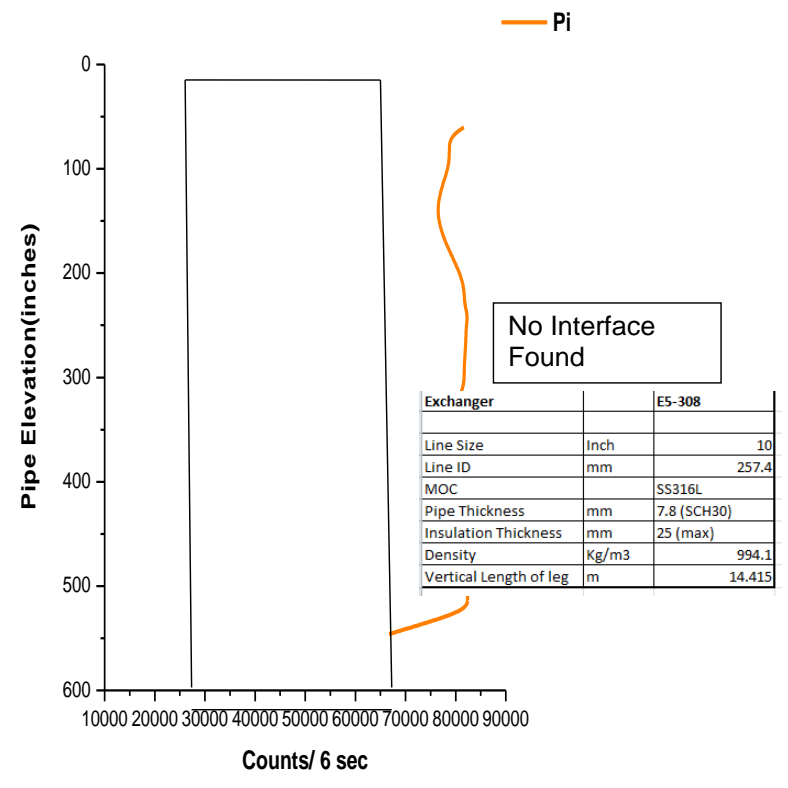

Fig 8: Interface in pipe 308

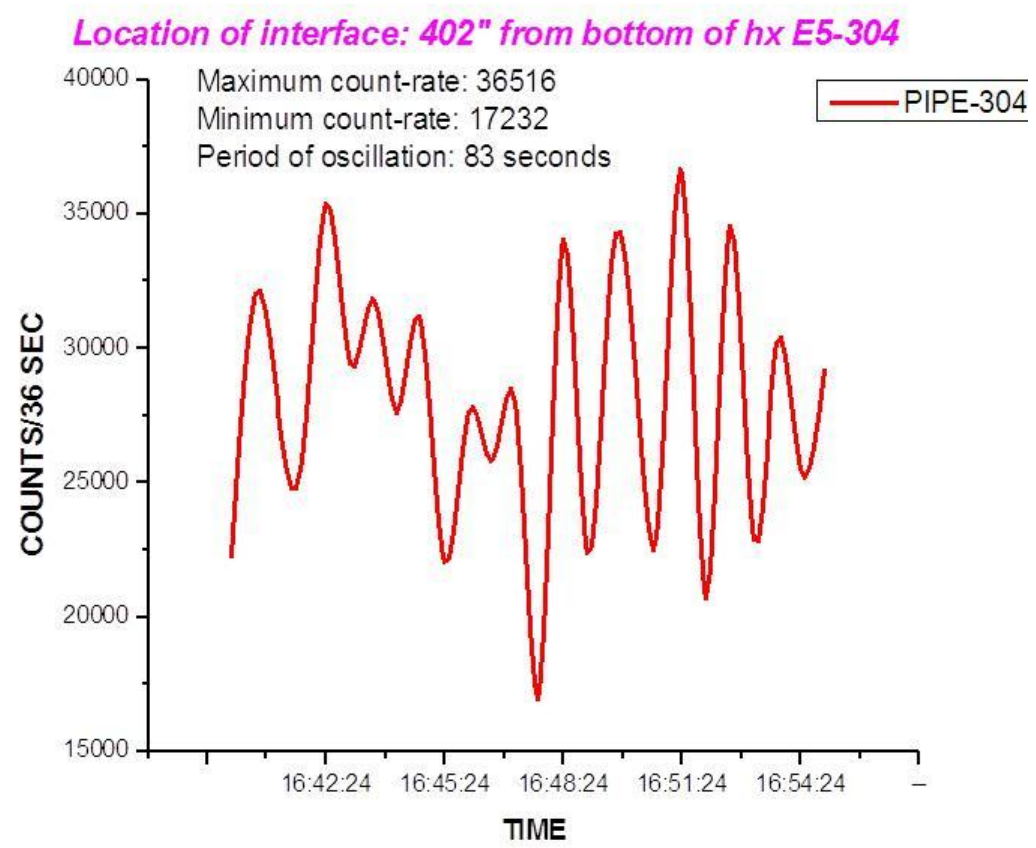

Fig 9: Period of oscillation in pipe E5-304:402" from bottom of HX 


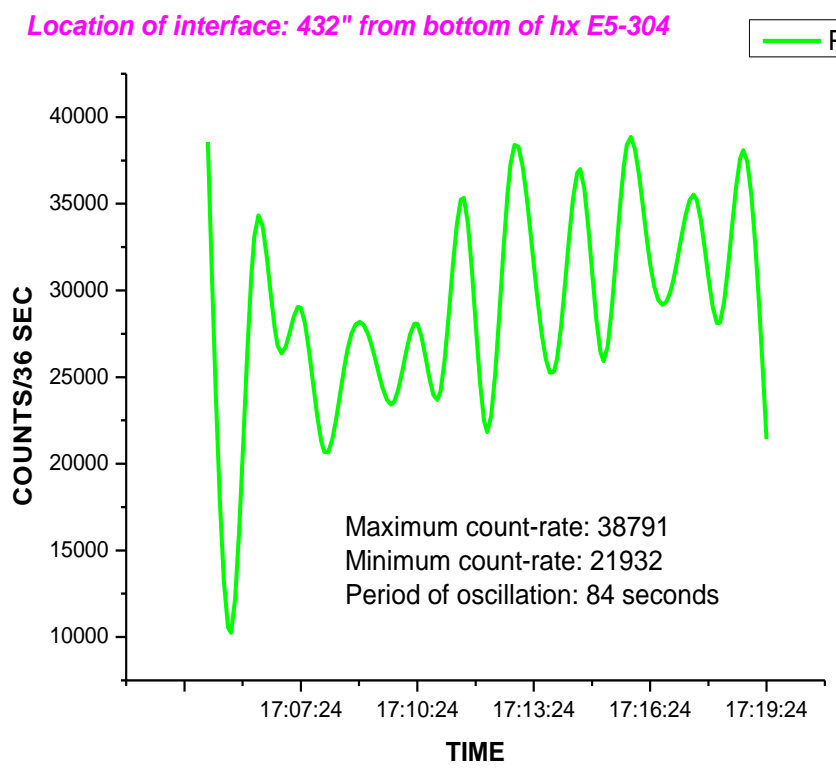

Fig 10: Period of oscillation in pipe E5-304:432"' from bottom of HX

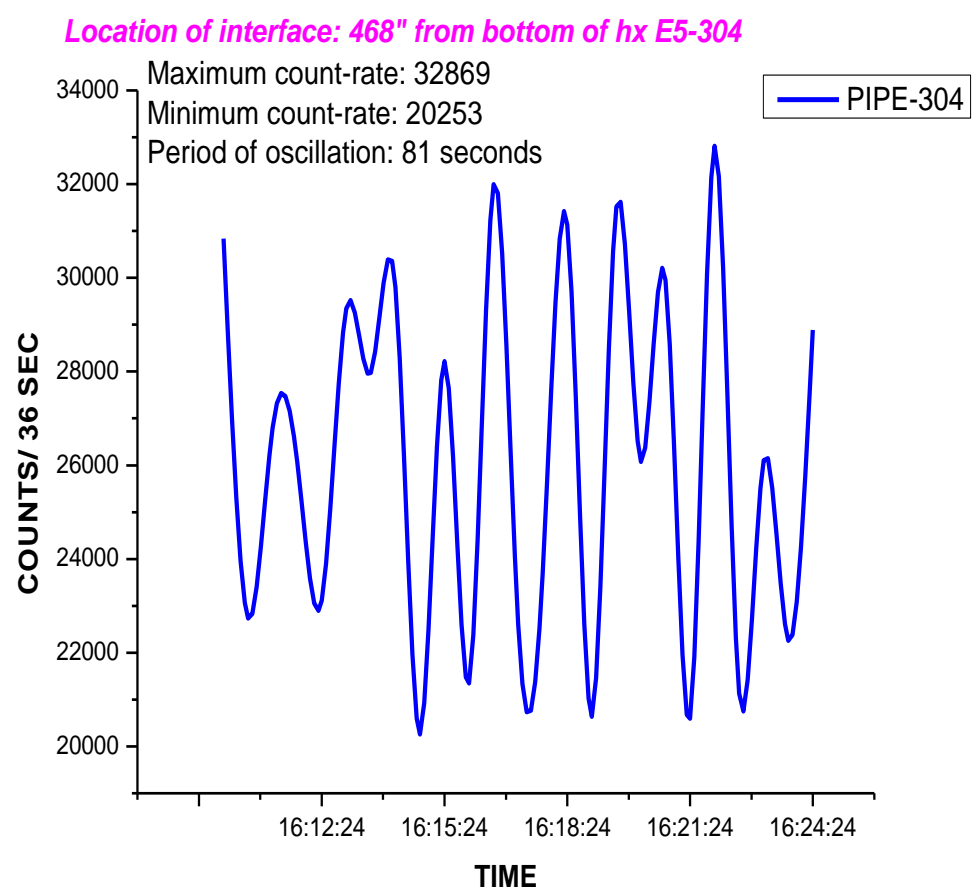

Fig 11: Period of oscillation in pipe E5-304:468', from bottom of HX 


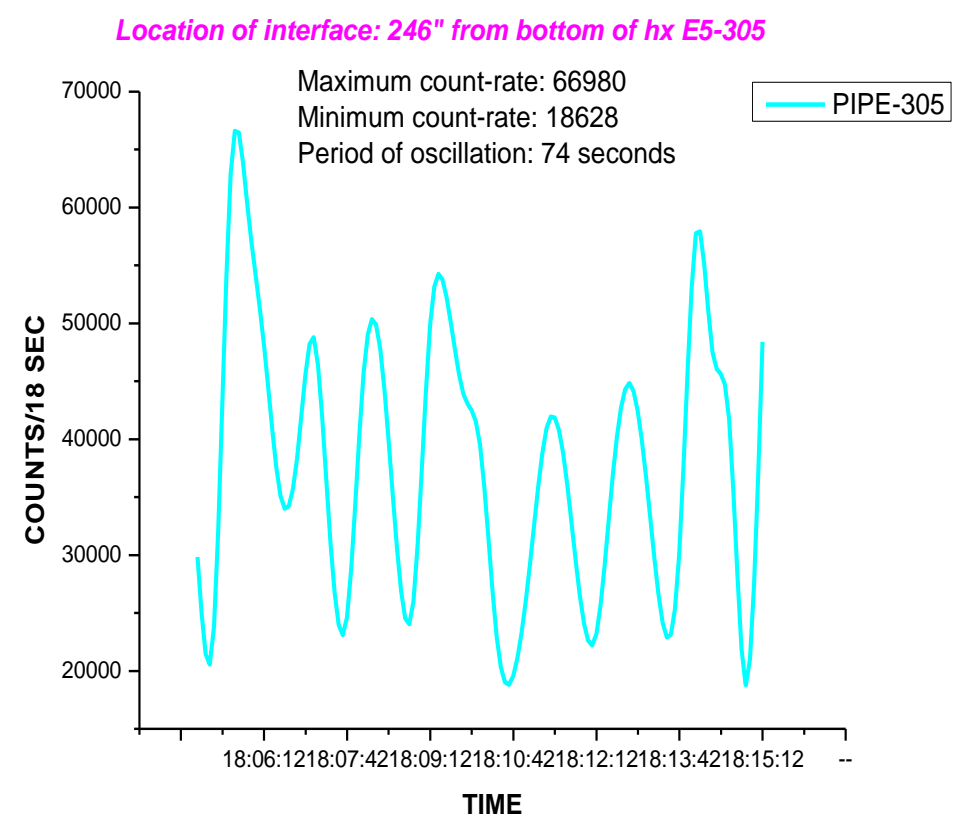

Fig 12: Period of oscillation in pipe E5-305:246" from bottom of $\mathrm{HX}$

Location of interface: 258 " from bottom of HX E5-306

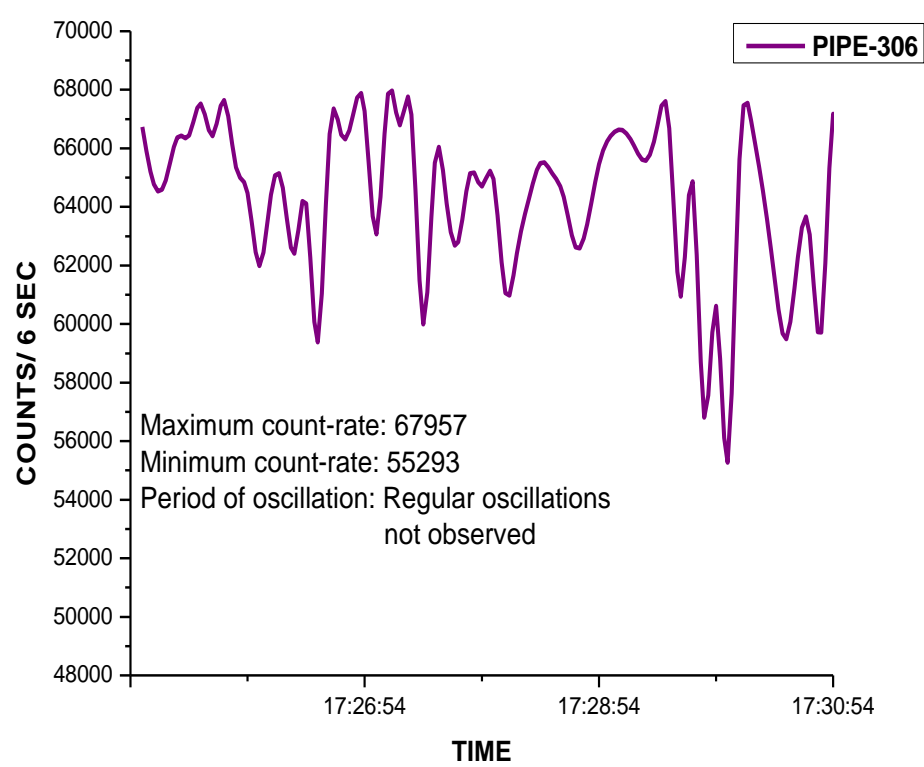

Fig 13: Period of oscillation in pipe E5-306:258', from bottom of HX 


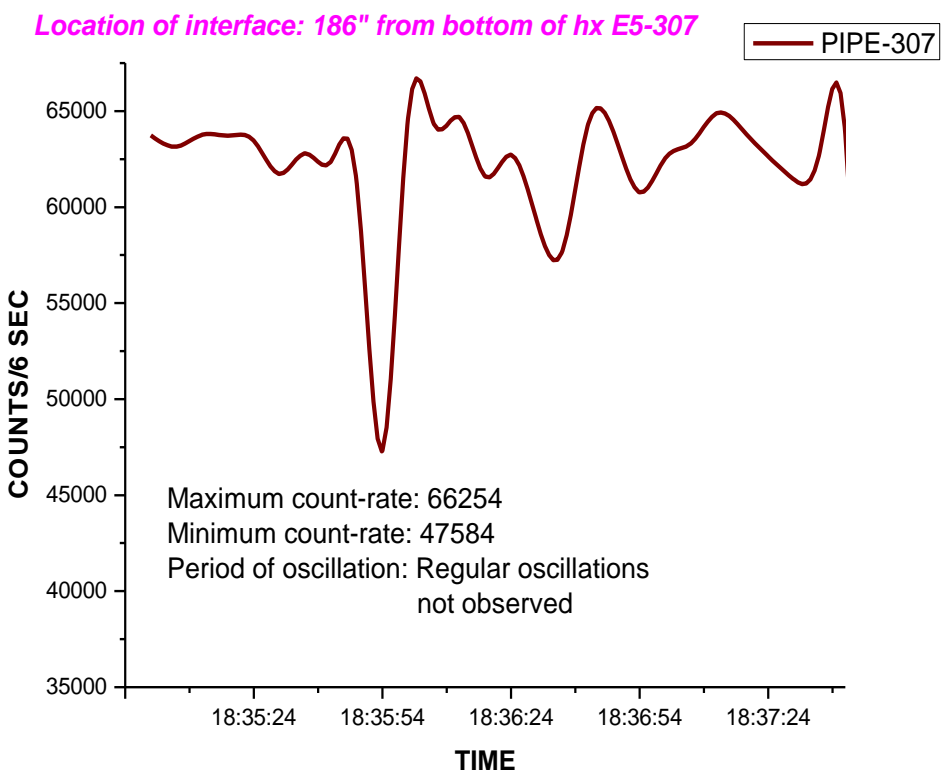

Fig 14: Period of oscillation in pipe E5-307:186" from bottom of HX

Location of interface: 60 " from bottom of HX E5-308

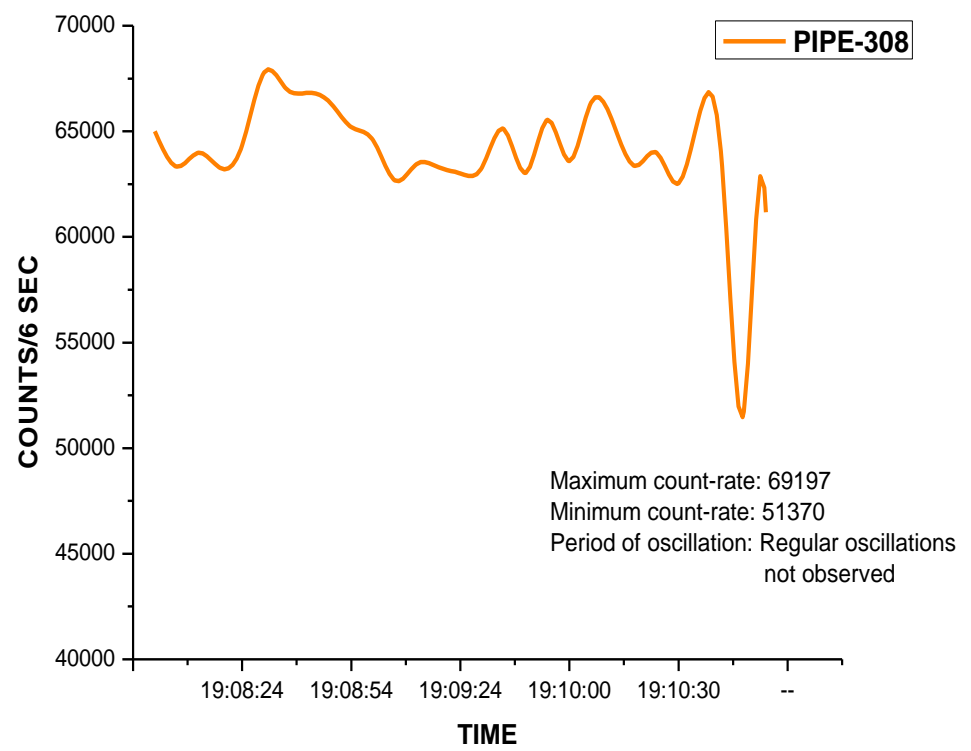

Fig 15: Period of oscillation in pipe E5-308:60" from bottom of HX 
Location of interface: 186" from bottom of HX E5-308

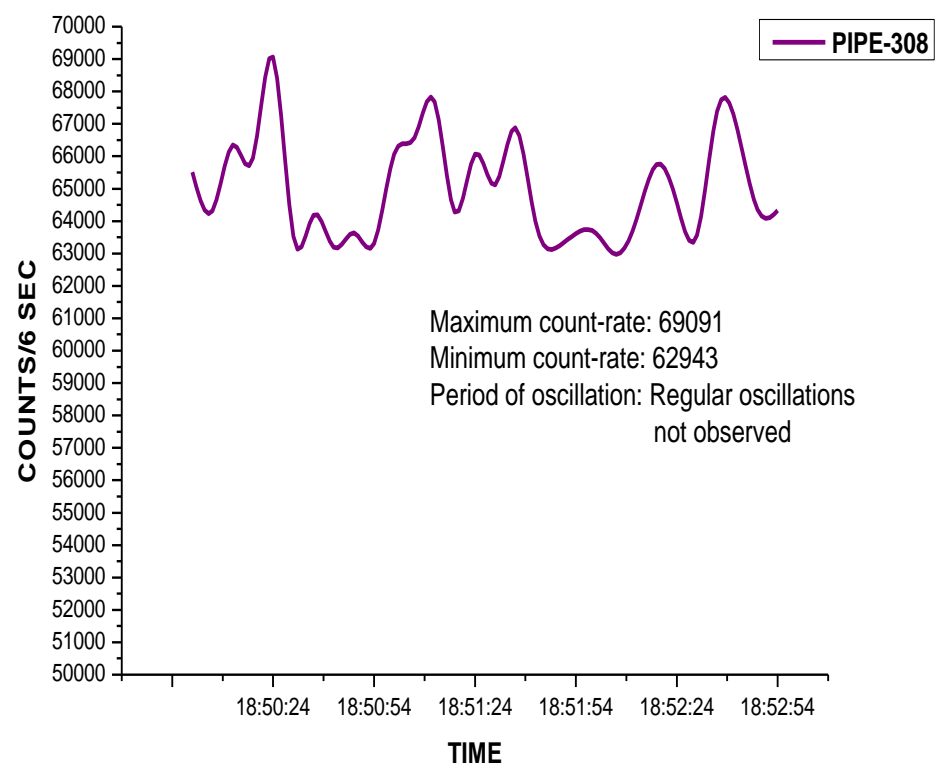

Fig 16: Period of oscillation in pipe E5-308:186"' from bottom of HX

\begin{tabular}{|r|c|c|}
\hline & B-2 & \\
\hline & T-JOINT & \\
\hline & UNINSULATED & \\
\hline & $\mathbf{X}$ & $\mathbf{Y}$ \\
\hline 1 & 10354 & 11365 \\
\hline 2 & 9875 & 12079 \\
\hline 3 & 9647 & 10441 \\
\hline 4 & 9019 & 8762 \\
\hline 5 & & 6328 \\
\hline 6 & & 6470 \\
\hline 7 & 7004 & 7058 \\
\hline 8 & 9190 & 10948 \\
\hline 9 & 9571 & 9932 \\
\hline 10 & 9325 & 9523 \\
\hline 11 & 8962 & 13250 \\
\hline 12 & 18430 & 13898 \\
\hline 13 & 12600 & 17822 \\
\hline 14 & 14272 & 14423 \\
\hline
\end{tabular}




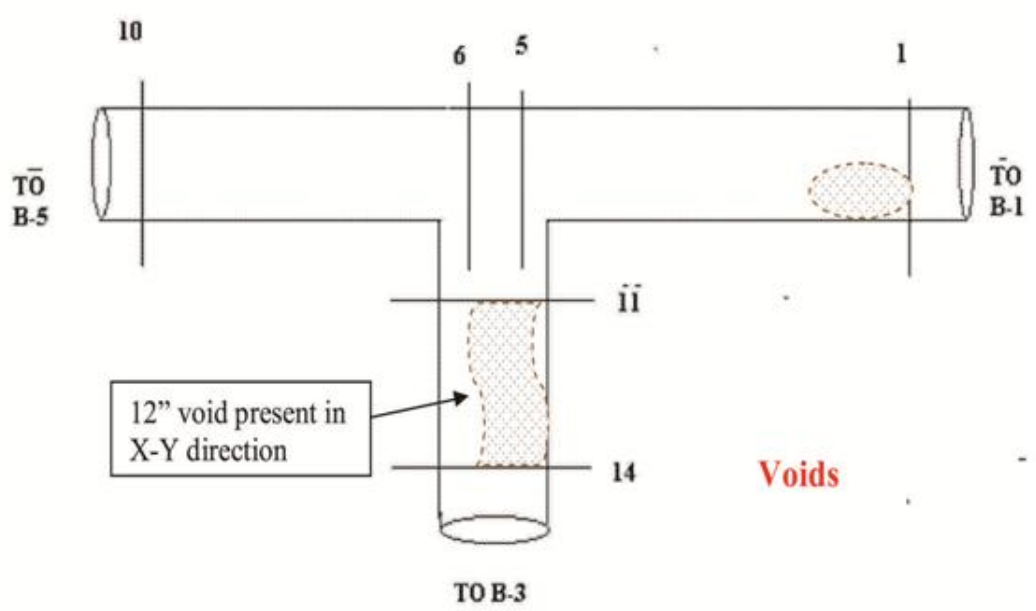

Fig 17: Voids in B-2 T-JOINT

\begin{tabular}{|c|c|c|c|}
\hline & $\begin{array}{l}\text { BEND } \\
\text { B-7 }\end{array}$ & & \\
\hline & $\mathbf{X}$ & $\mathbf{Y}$ & $\mathbf{Z}$ \\
\hline 1 & 31936 & 42306 & 54433 \\
\hline 2 & 30758 & 30973 & 32500 \\
\hline 3 & 35619 & 41206 & 32612 \\
\hline 4 & 30114 & 43024 & 31291 \\
\hline 5 & 42301 & 32283 & 34250 \\
\hline 6 & 37631 & 30217 & 30775 \\
\hline 7 & 35555 & 30467 & 23616 \\
\hline 8 & 30215 & 43480 & 15808 \\
\hline 9 & 28227 & 41319 & 15240 \\
\hline 10 & 19253 & 20622 & 18193 \\
\hline
\end{tabular}

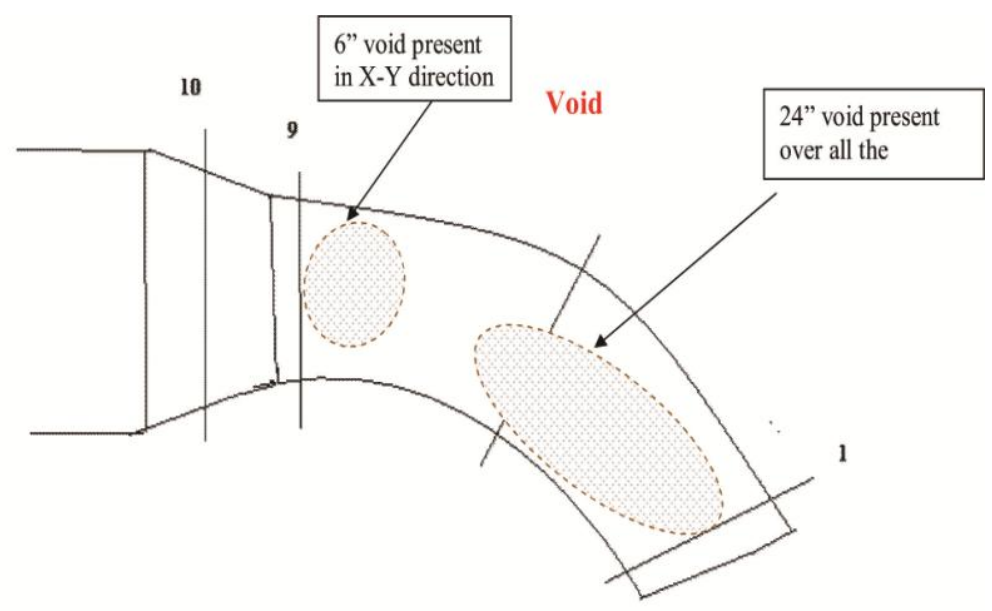

Fig 18: Voids in B-7 BEND 


\begin{tabular}{|c|c|c|c|}
\hline & \multirow{2}{*}{\multicolumn{2}{|c|}{\begin{tabular}{|l|l} 
B-10 & \\
INSULATED
\end{tabular}}} & \multirow[b]{3}{*}{$z$} \\
\hline & & & \\
\hline & $x$ & $Y$ & \\
\hline 1 & 18933 & 63052 & 26983 \\
\hline 2 & 68766 & 68256 & 25524 \\
\hline 3 & 29200 & 32798 & 66685 \\
\hline \begin{tabular}{|l|}
4 \\
\end{tabular} & 51798 & 15835 & 17674 \\
\hline 5 & 59605 & 21860 & 64343 \\
\hline 6 & 48585 & UNACCESIBLE & 52751 \\
\hline 7 & 20782 & UNACCESIBLE & 21801 \\
\hline
\end{tabular}

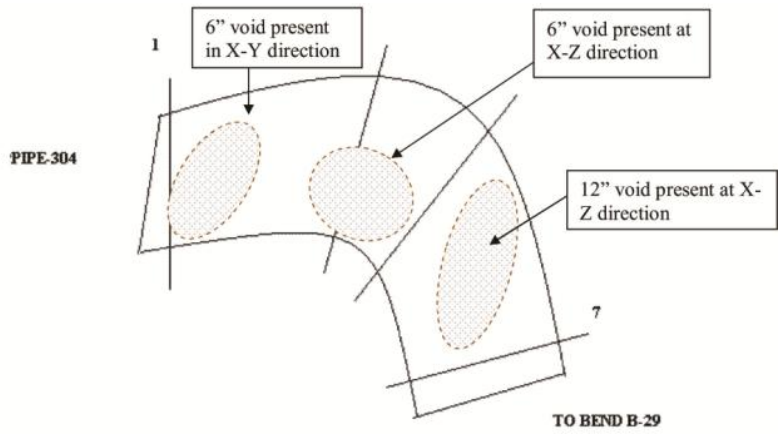

Fig 19: Voids in B-10 BEND

\begin{tabular}{|r|c|c|c|}
\hline & B-13 & & \\
\hline & INSULATED & & \\
\hline & $\mathbf{X}$ & $\mathbf{Y}$ & $\mathbf{Z}$ \\
\hline 1 & 2653 & 8136 & 1325 \\
\hline 2 & 4220 & 5058 & 4813 \\
\hline 3 & 1323 & 1971 & 1255 \\
\hline 4 & 874 & 4509 & 904 \\
\hline 5 & 2832 & 9927 & 2011 \\
\hline 6 & 2774 & 4321 & 1722 \\
\hline 7 & 6102 & 1880 & 1290 \\
\hline 8 & 1733 & 2878 & 627 \\
\hline 9 & 1415 & 4699 & 479 \\
\hline 10 & 902 & 7570 & 989 \\
\hline 11 & 1597 & 4423 & 2149 \\
\hline 12 & 2001 & 1733 & 2977 \\
\hline 13 & 5663 & 6180 & 2534 \\
\hline 14 & 1845 & 4281 & 2843 \\
\hline 15 & 1368 & 2426 & 1911 \\
\hline 16 & 987 & 2163 & 3816 \\
\hline 17 & 2755 & 3044 & 4487 \\
\hline 18 & 5096 & 7181 & 4863 \\
\hline 19 & 2556 & 3467 & 4892 \\
\hline
\end{tabular}

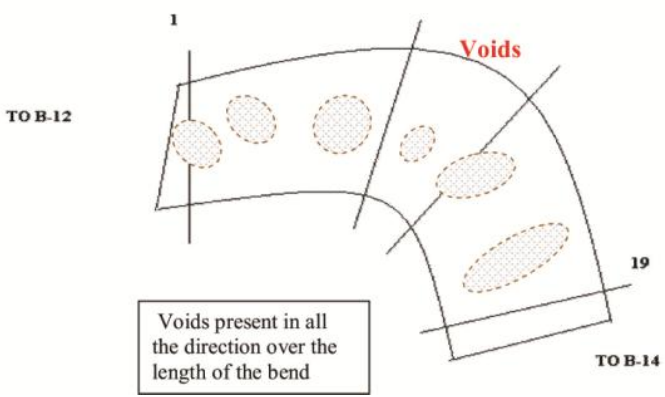

Fig 20: Voids in B-13 BEND 


\begin{tabular}{|r|c|c|c|}
\hline & B-14 & & \\
\hline & T-JOINT & & \\
\hline & $\mathbf{X}$ & $\mathbf{Y}$ & $\mathbf{Z}$ \\
\hline 1 & 14309 & 3171 & 1370 \\
\hline 2 & 1075 & 2203 & 1782 \\
\hline 3 & 1521 & & 973 \\
\hline 4 & 1536 & & 1355 \\
\hline 5 & 3870 & 14106 & 2121 \\
\hline 6 & 6051 & 10155 & 1810 \\
\hline 7 & 7225 & 7416 & 1965 \\
\hline 8 & 25830 & 10184 & 2044 \\
\hline 9 & 27784 & 25384 & 28972 \\
\hline 10 & 24368 & 29581 & 26499 \\
\hline
\end{tabular}

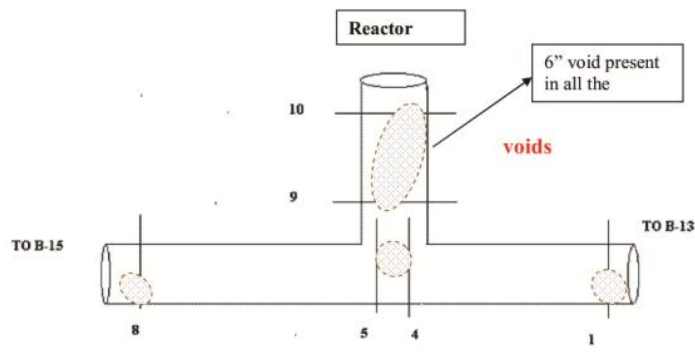

Fig 21: Voids in B-14 T-JOINT

\begin{tabular}{|r|r|r|r|}
\hline & B-19B & & \\
\hline & BEND & & \\
\hline & X & \multicolumn{1}{l|}{$\mathbf{Y}$} & \multicolumn{1}{l|}{} \\
\hline 1 & 3236 & 7760 & 4701 \\
\hline 2 & 4153 & 6463 & 4859 \\
\hline 3 & 4440 & 11653 & 5260 \\
\hline 4 & 4154 & 7567 & 5908 \\
\hline 5 & 4391 & 6002 & 6393 \\
\hline 6 & 4830 & 5358 & 7790 \\
\hline 7 & 4934 & 6218 & 12135 \\
\hline 8 & 4095 & 11678 & 7863 \\
\hline 9 & 4250 & 11013 & 5449 \\
\hline 10 & 3864 & 7882 & 6513 \\
\hline 12 & 4060 & 8435 & 4830 \\
\hline 13 & 5411 & 7984 & 13343 \\
\hline
\end{tabular}

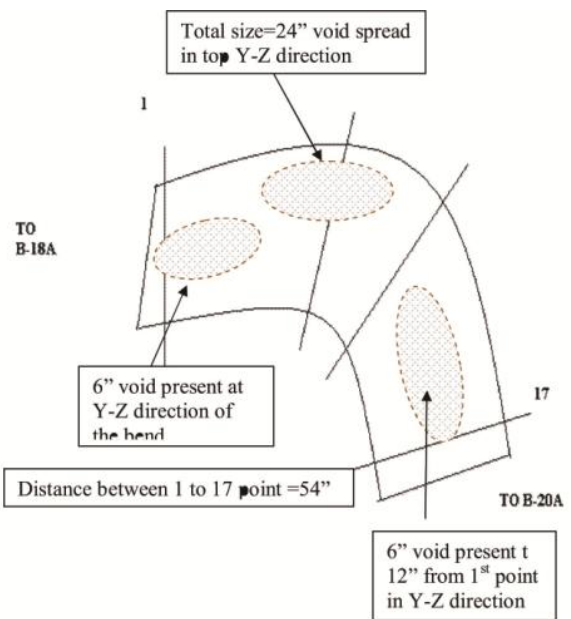

Fig 22: Voids in B-19 B BEND 


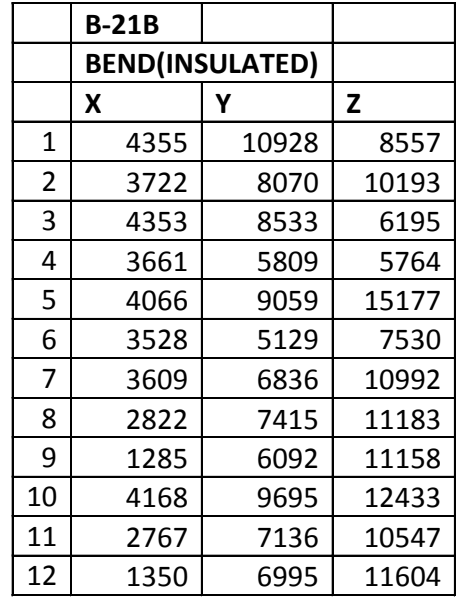

Voids

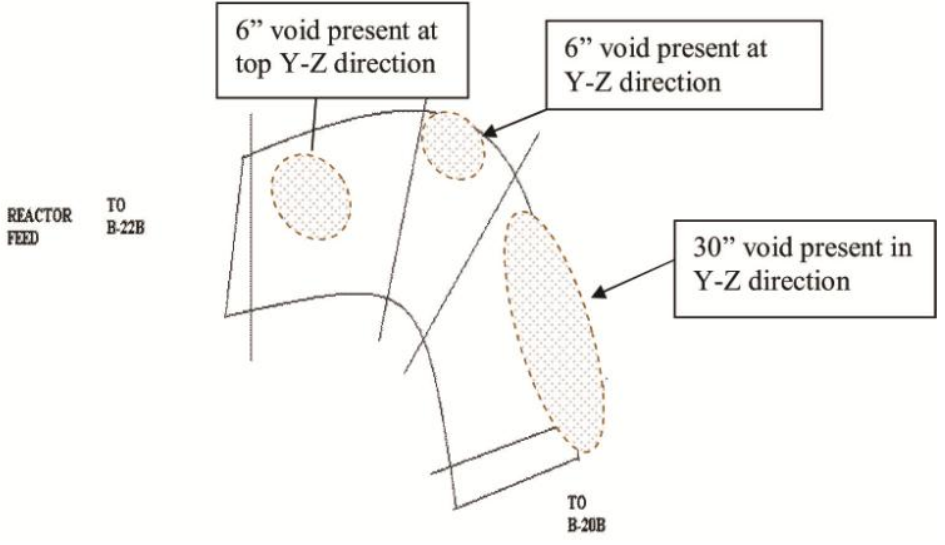

Fig 23: Voids in B-21B BEND 